\title{
Surgical Treatment of Ten Adults with Spinal Extradural Meningeal Cysts in the Thoracolumbar Spine
}

\author{
Feifan $\mathrm{Xu}{ }^{1,2}$ Fengzeng Jian, ${ }^{2}$ Liang $\mathrm{Li},{ }^{1}$ Jian Guan, ${ }^{2}$ Zan Chen $^{2}$ \\ Department of Neurosurgery, Peking University First Hospital, Beijing, China \\ Department of Neurosurgery, ${ }^{2}$ Xuanwu Hospital, Capital Medical University, Beijing, China
}

Objective : To retrospectively analyze the clinical characteristics and surgical experience of 10 adults with spinal extradural meningeal cysts (SEMCS) in the thoracolumbar spine which may further provide evidence for surgical decision-making.

Methods : Ten adults with SEMCs in the thoracolumbar spine were surgically treated and enrolled in this study. Clinical manifestations, imaging data, intraoperative findings and postoperative outcome were recorded.

Results : Clinical manifestations of SEMCs included motor and sensory dysfunction of the lower limbs and urination and defecation disturbance. The cysts presented as intraspinal occupying lesions dorsal to the spine, ranging from the T8 to L3 level. Defects of eight cases were found on preoperative magnetic resonance imaging (MRI). Selective hemilaminectomy or laminectomy were used to reveal the defect within the cyst, which was further sutured with microscopic technique. The final outcome was excellent or good in seven cases and fair in three cases. No recurrence was observed during follow-up.

Conclusion : SEMCs are rare intraspinal cystic lesions. Radiography and MRI are clinically practical methods to assess defects within SEMCs. Selective hemilaminectomy or laminectomy may reduce surgical trauma. Detection and microscopic suturing of the defects are the key steps to adequately decompress the nervous tissue and prevent postoperative recurrence.

Key Words : Spinal extradural meningeal cysts · Dural defect · Dural diverticula · Surgery - Thoracolumbar.

\section{INTRODUCTION}

Spinal extradural meningeal cysts (SEMCs) are rare pathological entities caused by intraspinal cystic compression to the spinal cord and/or nerve roots which may lead to a series of neurological disorders, accounting for only $1-3 \%$ of intraspinal lesions ${ }^{1,1,24)}$. As the pathogenesis is not clear, many nomenclatures were used in the previous reports to describe these entities, such as extradural meningeal/arachnoid cysts, arach- noid diverticula, pouches, meningoceles ${ }^{1,19,20,22,27)}$. Most SEMCs are usually found at dorsal or dorsolateral side of the spinal cord and are especially common at the thoracic or thoracolumbar segments in adults, resulting in sensory-motor disturbance or sphincter dysfunction ${ }^{5,24)}$. In this study, we retrospectively analyzed clinical characteristics, location of defects and surgical experience of ten adults with SEMCs in the thoracolumbar spine which may further provide evidence for surgical decision-making.

- Received : August 21, 2020 •Revised : September 14, 2020 •Accepted : October 13, 2020

- Address for reprints : Zan Chen

Department of Neurosurgery, Xuanwu Hospital, Capital Medical University, China-INI, 45 Changchun Street, Beijing 100053, China

Tel : +86-18811176550, Fax : +86-010-83192622, E-mail : neurospine66@163.com, ORCID : https://orcid.org/0000-0002-7208-4149

This is an Open Access article distributed under the terms of the Creative Commons Attribution Non-Commercial License (http://creativecommons.org/licenses/by-nc/4.0) which permits unrestricted non-commercial use, distribution, and reproduction in any medium, provided the original work is properly cited. 


\section{MATERIALS AND METHODS}

\section{General information}

All procedures performed in this study were in accordance with the ethical committee of Xuanwu Hospital, Capital Medical University. Informed consent was obtained for all enrolled patients. From January 2017 to November 2019, ten adults with single symptomatic SEMC in the thoracolumbar spine were admitted to our hospital and received surgical treatment. The patients' clinical manifestation, physical examination and imaging data were carefully recorded prior to analysis.

\section{Assessment of defect location}

Radiography and magnetic resonance imaging (MRI) were the two main methods for the assessment of dural defects. Assessment criteria : a possible location of a defect might be indicated by severe bony erosion in the vertebral body and/or pedicle, significant enlarged intervertebral foramen on radiography, or a regional expanded cyst wall which usually protrudes to one

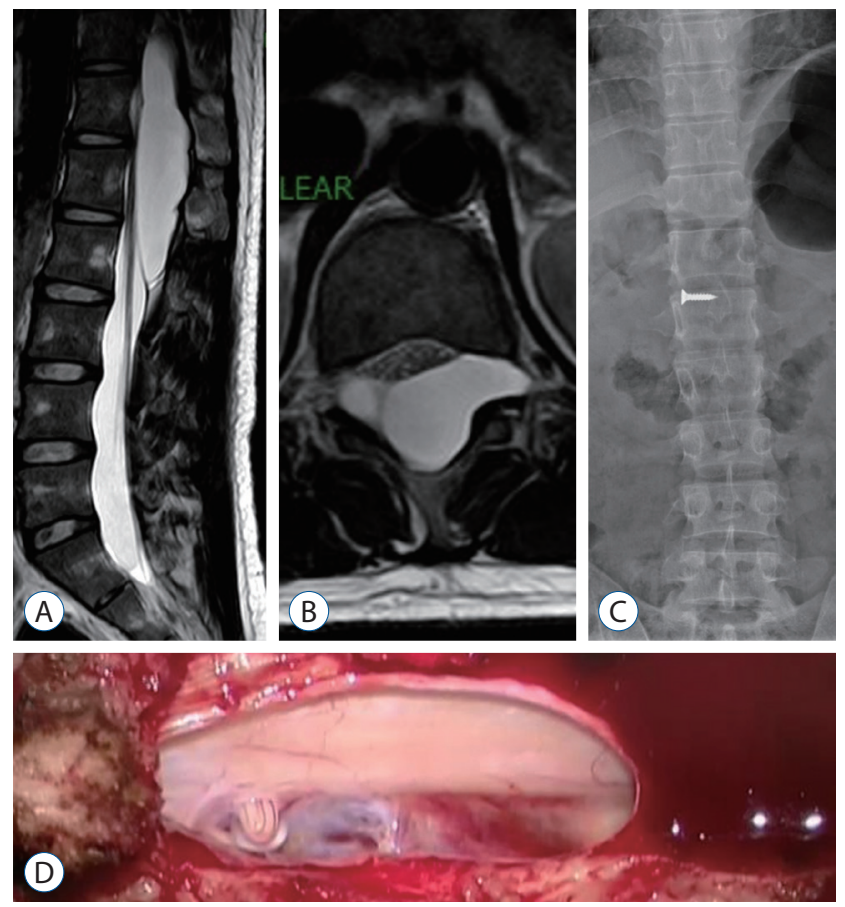

Fig. 1. Case No. 12. A : Sagittal T2-weighted MR image demonstrates a cyst extending from $T 11$ to $L 2$ with dorsal compression of the spinal cord and cauda equina. B : Axial T2-weighted image reveals cyst extension through the left neural foramina. $C$ : Anteroposterior radiography shows narrowing of bilateral vertebral pedicles from T12 to L1 (nail pointed to spinous process of T12). D : Nerve root fiber moves back and forth via the dural defect. side on the horizontal MR image (Figs. 1 and 2) ${ }^{12,13}$. Some T2weighted MR images revealed a lower signal within the cyst, which could be the result of flow voids caused by cerebrospinal fluid (CSF) going through the dural defect (Fig. 3).

\section{Surgical management}

A posterior approach was performed within the field containing the SEMC and defect according to preoperative radiography and MRI. Selective hemilaminectomy or laminotomy was used to remove lamina where the defect of the SEMC would possibly be. Exposure of the caudal or rostral boundary of the SEMC was unnecessary and should be avoided. The outer cyst wall was incised longitudinally, yielding CSF-like fluid, and the inner cyst wall was examined through the cyst under microscope. After revealing the defect, $8-0$ nylon suture was used to repair it with the microsurgical technique. Muscle mud and fibrin sealant were applied to strengthen the sutured defect as necessary (Fig. 3). If the defect was not found regionally, a further exploration would be applied upwards or downwards, or on the contralateral side along the incision. The outer wall of the cyst was left open.
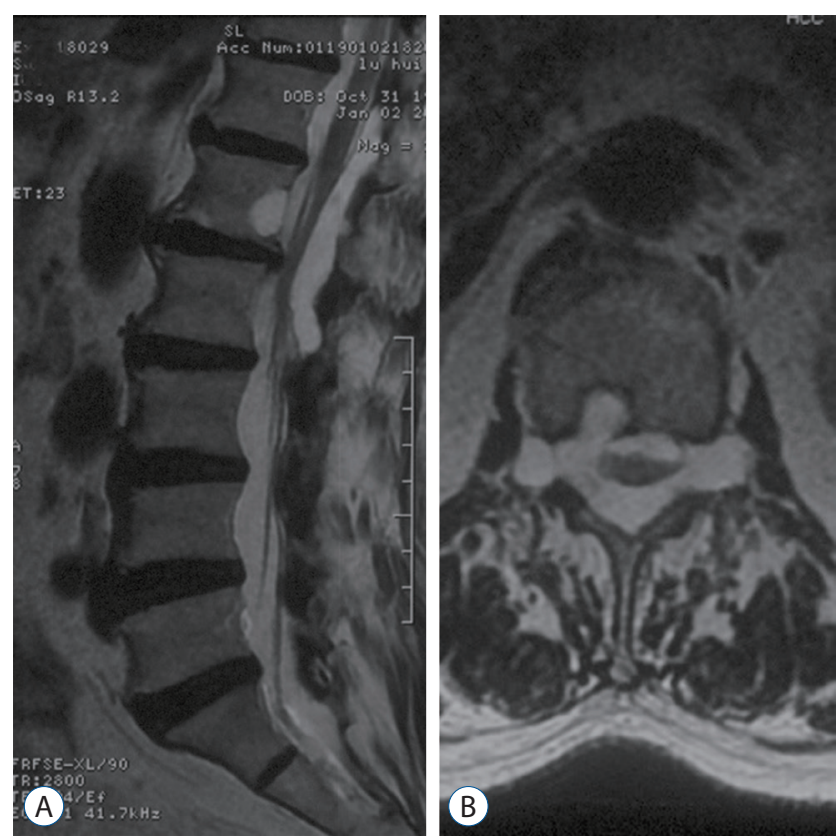

Fig. 2. Case No. 7. A : Sagittal T2-weighted magnetic resonance image demonstrates an irregular cyst extending from T12 to L2 with dorsal compression of the spinal cord. B : Axial T2-weighted image shows a bony erosion in the back of L1 vertebral body (right side) by spinal extradural meningeal cyst and enlargement of bilateral intervertebral foramens. 

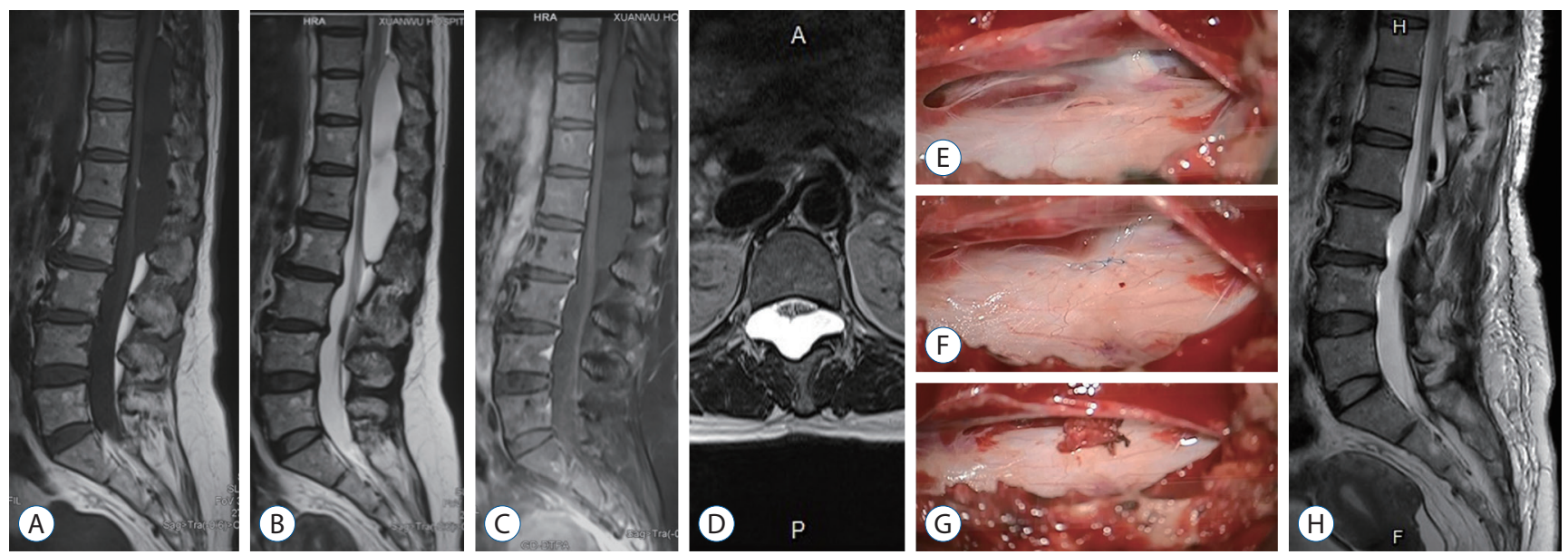

Fig. 3. Case No. 3. A-C : A cyst extended from T10 to L2 with dorsal compression of the spinal cord. All SEMCs shows homogenous low-intensity signals on T1-weighted MRI and high-intensity signals on T2-weighted MRI. No enhancement is demonstrated by contrast agents. A suspected flow void is identified at T12 level. D : Axial T2-weighted image (T12 level) reveals the cyst is larger in the right side. E : Dural defect is identified under T12 nerve root sleeve during surgery, and the nerve root is adherent to the defect. F : Closing of the defect. G : Strengthening with muscle and fibrin sealant at the defect. $\mathrm{H}$ : Significant resolution of SEMC and nerve decompression after surgery. SEMC : spinal extradural meningeal cyst, MRI : magnetic resonance imaging.

\section{Outcome and follow-up}

Postoperatively, patients were regularly followed-up after discharge at 3 months, 6 months, 1 year, and 2 years, including MRI, outpatient examination and telephone interviews. The surgical outcome was evaluated on a four-grade scale as previously described $^{23)}: 1$ ) excellent (no pain and no neurological deficits); 2) good (occasional mild pain and no neurological deficits); 3) fair (frequent pain, slightly improved neurological deficits, and some change in work status); and 4) poor (disabling pain, unchanged neurological deficit, and unable to work).

\section{RESULTS}

\section{Clinical presentations}

General information of our series is displayed in Table 1. The average age of the ten patients was 47.7 years (range, 2480). The average duration from onset to surgery was 86.1 months (range, 1-300). The male-to-female ratio was $1: 1$. Five patients exhibited motor disorders of the legs (including three patients with bilateral lower limb weakness and two patients with unilateral lower limb weakness, one patient with amyotrophy), six exhibited sensory disorders (including five patients with waist ache and four patients with numbness of two legs). Load-bearing activity, sitting for a long time, and coughing aggravated symptoms, but most patients were relieved after rest. Only one patient complained of long-term urination and defecation disturbance. No asymptomatic patients were enrolled in this study.

\section{Radiological findings}

All SEMCs showed the same radiological characteristics as CSF on MRI : low-intensity signals on T1-weighted images, high-intensity signals on T2-weighted images, and no enhancement displayed by contrast agents (Fig. 3). The ten cysts presented as occupying lesions dorsal to the spinal cord and cauda equina and centered at the thoracolumbar segments (range, T8 to L3 level). The average length was 3.9 spinal segments (range, 2-7). According to our preoperative imaging criteria for assessment, of the 10 cases, eight patients presented with clear defects (include segment and/or side), while the other two cases' defects were ambiguous (Table 1).

\section{Surgical findings}

All patients underwent microscopic surgeries, which were performed by a single senior surgeon. Hemilaminectomy (six cases) or laminectomy (four cases) were applied according to the defect position predicated with preoperative MRI and radiography (Table 1; range, T11 to L2 spinal segment). During surgery, all cysts with very thin walls were identified dorsal to the spinal cord and cauda equina. There was only one small 


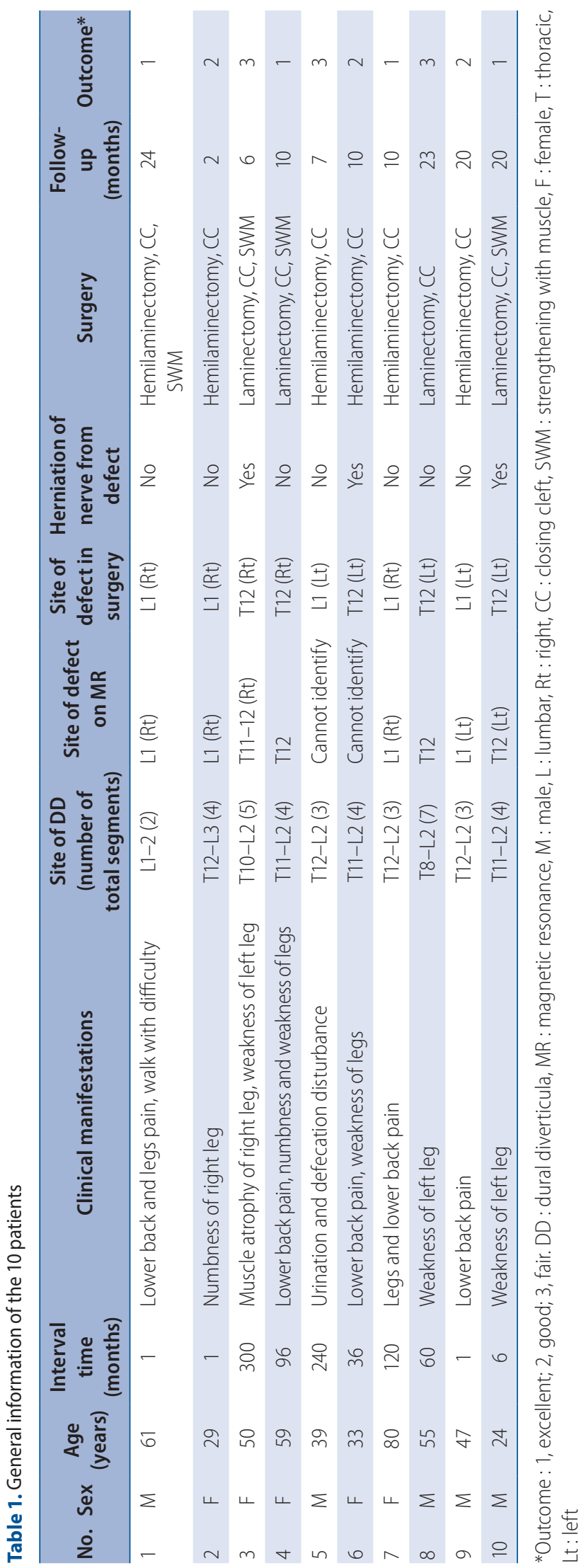

longitudinal or oval defect with smooth margin confirmed in each SEMC (range, approximately 3 to $10 \mathrm{~mm}$ in length) and they were all located near the nerve root sleeve (five around L1 and the other five near the T12 nerve root sleeve). CSF flowed out from the defects regularly, which theoretically result in the expansion of the SEMCs. The left-to-right side ratio of defects was $5: 5$. SEMCs connected with subarachnoid space via dural defects, under which spinal cords or nerve roots adherent to the defects could be revealed (Fig. 3). Nerve root fibers moved back and forth with a CSF pulse through the defect in four patients. All defects were fully repaired during microscopic surgery after returning nerve root fibers to the subarachnoid space as necessary. Four sutured defects were strengthened with muscle mud and fibrin sealant to avoid CSF leakage. Complete cyst excision was not applied.

\section{Neuropathology}

Postoperative neuropathological analysis of resected cyst wall demonstrated arachnoid epithelial and compact fibrous connective tissue.

\section{Outcome and follow-up}

The average follow-up duration was 13.2 months (range, 2-24). On the four-grade scale, the postoperative outcome was excellent in four cases, good in three, fair in three and poor in none. The postoperative courses of all patients were uneventful and none experienced remarkable perioperative complications. Residual cysts shrank dramatically or even completely disappeared. Decompression of the spinal cord and cauda equina was fully achieved at follow-up with no recurrence on MRI.

\section{DISCUSSION}

\section{Etiology}

In previous literature, the classifications for pathologies of spinal meninges are controversial. In 1988, Nabors et al. ${ }^{20)}$ divided spinal meningeal cysts into three categories : type I, cysts without nerve root fibers (subdivided into IA, extradural arachnoid cysts, and IB, sacral meningoceles); type II, cysts with nerve root fibers; and type III, intradural meningeal cysts. Recently in 2017, Klekamp ${ }^{11}$ indicated that the classification by Nabors et al. ${ }^{20)}$ was not accurate and comprehensive 
in terms of different pathologies of spinal meninges. Therefore, he introduced a new classification system which may represent distinct entities requiring different approaches for diagnosis and treatment. Pathologies of the spinal dura were classified into three categories : dural diverticula (type I), dissections (type II), and ectasias (type III), and SEMCs were categorized as dural diverticula.

SEMCs presented as extradural cystic occupying lesions filled by $\mathrm{CSF}^{11)}$. The mainstream hypothesis is that SEMC is caused by herniation of arachnoid tissue via a dural defect which connecting the cyst and the subarachnoid space. The cyst gradually expands with tension as CSF gathers within it and finally compresses the nervous tissues ${ }^{1,14,24)}$. Postoperative histopathology of the resected cyst wall revealed arachnoid epithelial and compact fibrous connective tissue, which suggested dural components are contained in the outer wall of the cyst ${ }^{1,2,11,26)}$.

Suspected reasons for cyst expansion are still debatable, including the osmotic pressure gradient between the cyst and the subarachnoid space, CSF pulsation and high hydrostatic pressure, and arachnoid secretion theory ${ }^{3,22,24)}$. Among them, many researchers agree with the valve-like mechanism : the dural defect works as a ball valve, creating a two-way system of unequal CSF flow, allowing CSF to move intermittently from the high-pressure subarachnoid space to the low-pressure cyst, thus resulting in cyst enlargement ${ }^{2,13)}$. Morizane et al. ${ }^{19)}$ found in seven patients that nerve root fiber moved back and forth around the dural defects that allowed CSF to flow into the cysts, which may be considered as a new valve-like mechanism.

\section{Location of defects and intraoperative findings}

One difficulty for surgical management of SEMC is the detection for the dural defect. An accurate preoperative assessment of the defect plays an important role in formulating surgical strategy. Previous reports introduced several methods for location, including cine-MRI, computed tomography myelography, time-spatial labeling inversion pulse MRI, digital subtraction cystography and MR myelography ${ }^{6,8,9,16,18,21)}$. However, due to sporadic reports of cases for each examination and a frequent occurrence of negative results, no single method is significantly better than the others.

Based on our experience and previous literature, a defect of thoracolumbar SEMC is commonly found at the T12 to L1 segments, in close proximity to the branching point of the nerve root sleeve $e^{1,3,11,12)}$. In our series, all 10 defects were located in this region. Suspected causes include : 1) a larger range of motion in this area facilitates tearing of the inner layer near the nerve root sleeve; and 2) this area is vulnerable to congenital dural hypoplasia ${ }^{1)}$. As most defects are located to one side of nerve root sleeve, the walls of cysts at defects are likely to bulge and protrude laterally and show regional flow-voids on MRI. The formation of SEMCs are slow, and they occupy space. They can gradually destroy the bony structure of spine, usually presenting as erosive changes in the vertebral body, pedicle and foramen, which can be used as indirect but reliable imaging evidence to identify the position of the defect. In our series, the locations of defects in all eight patients whose defects were identified preoperatively were certified during surgery, which means that as long as the assessment criteria is rational, X-ray plain film and MRI are enough to effectively estimate the dural defects. For defects that are hard to identify, we suggest starting surgical exploration from the T12-L1 segments. If the laterality of the defect is uncertain, selective laminectomy is recommended in order to expose the bilateral nerve root sleeves at the same time.

\section{Treatment strategy and surgical outcome}

Microinvasive surgery is preferred by most authors as the first choice for management ${ }^{25)}$. However, there is still no agreement on the most effective surgical strategies. Grag et al. ${ }^{5)}$ reported ten cases of SEMCs who underwent unselective laminoplasty and total resections which covered the whole range of cysts. Among them, dural defects were found and repaired in eight patients, and cyst resections were performed in nine patients, except for one patient in whom the cyst expanded from C3 to L2 and marsupialization was applied ${ }^{5)}$. Funao et al. ${ }^{3)}$ divided 12 thoracolumbar SEMCs patients into two groups : in the first group undergoing total resection of the cyst (seven cases), unselective laminectomy was performed at all the levels where the cyst existed; whereas in the second group undergoing closure of the dural defect without cyst resection (five cases), they performed selective laminectomy only at the level of the communication site preoperatively identified by neuroimaging and detected the defect by inserting a flexible neuroendoscope into the cyst cavity. In terms of the functional recovery, they did not observe a significant difference between the two groups, but the second surgical pro- 
cedure was less invasive and better at reducing the mean postoperative kyphotic angle of the thoracolumbar spine.

Laminectomy needs to separate bilateral paravertebral muscles and open the spinal process and lamina complex. As the exposed segments increase, massive surgical trauma is unavoidable ${ }^{1723)}$. Multiple-level laminectomy can destroy the balance of spinal stability, which may finally induce kyphosis in surgical thoracolumbar segments. Hemilaminectomy is more microinvasive to keep the spinal process and lamina intact on the contralateral side. It is highly advantageous for the protection of spinal stability, but higher requirements are needed, such as accurate preoperative identification of the dural defect and experienced microsurgical skills (Fig. 4) ${ }^{13)}$. In addition, we recommend a selective surgical approach ${ }^{12)}$. Selective hemilaminectomy (with identified spinal segment and laterality) or laminectomy (with identified segment) is preferred as long as the position of the defect is initially confirmed before surgery $^{13)}$. If the defect is not detected in the selective region under microscope, further exploration could be performed upwards or downwards or on the other side along the incision. Because multiple-segment laminectomy tends to induce vertebral deformation as a long-term postoperative complication, fusion and fixation in regional thoracolumbar segments are suggested $^{3-5)}$.
Defect repairment and total cyst excision are considered by most authors as the mainstream management method, but the best surgical treatment is still debatable $e^{12,24,26}$. Hatashita et al. ${ }^{7)}$ suggested that defect repair is not necessary as long as total cyst resection is achieved. This idea is mainly based on some cases without detectable defects within cysts, or postoperative recurrence after total cyst excision. After analyzing their own surgical experience (eight patients) and previous reports, Lee et al. ${ }^{12)}$ demonstrated that there was no significantly positive relationship between total cyst excision and postoperative outcome or recurrence rate. But larger surgical exposure tended to increase the risk of complications after surgery. On the contrary, patients with defect repair have significantly better postoperative outcomes and lower in recurrence rates compared with patients without defect closure.

The purposes of surgery for SEMCs include two key points : 1) to relieve compression by the cyst on the spinal cord and nerve root in order to restore neurological function and 2) to close the defect between the cyst and subarachnoid space to prevent recurrence ${ }^{24)}$. The core pathogenesis of SEMC is the long-term and consistent impact of CSF flow to the dural defect, which leads to an expansion of the cyst. Therefore, defect repair is the most crucial treatment to prevent postoperative recurrence ${ }^{1,11,19,25,27)}$. In this study, our surgical strategy focused
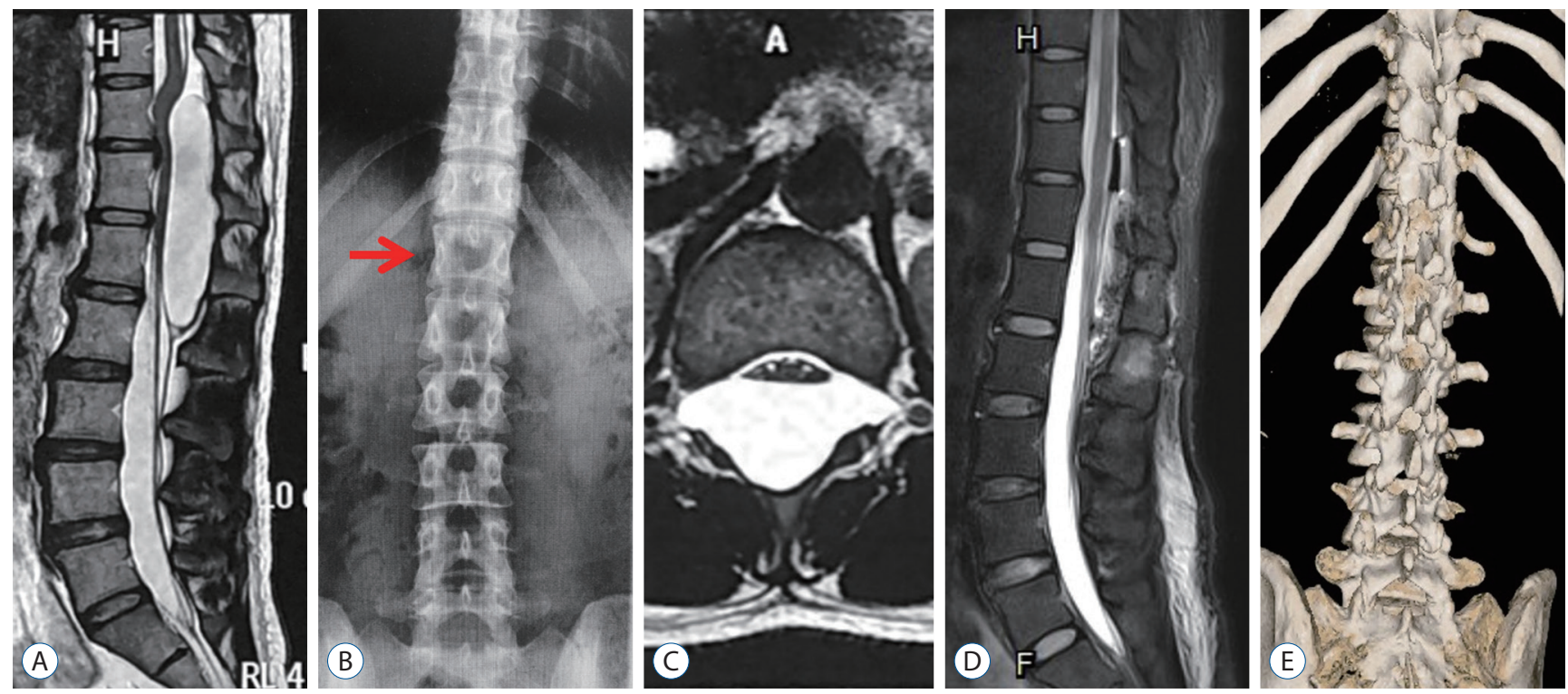

Fig. 4. Case No. 6. A : A cyst extends from $T 11$ to $L 2$ segment. A suspected flow void is identified at $T 12$ level. $B$ : Anteroposterior radiogram demonstrates significant narrowing of left vertebral pedicles of T12 (red arrow). C : However, the cyst presents symmetrically at T12 level on axial T2-weighted image. D : The cyst shranks dramatically and nerve decompression is achieved. E : Postoperative three-dimensional computed tomography reconstruction illustrates the range of hemilaminectomy (from T12 to L2). 
on detecting and tightly repairing dural defects in all ten patients, without total cyst excision. This strategy has several advantages $\left.^{15,24)}: 1\right)$ to avoid increasing the risk of postoperative kyphosis due to extensive removal of vertebral plates; 2) to block the formation and expansion of SEMCs by aiming at the primary cause; and 3) to reduce recurrence rate. As hemilaminectomy was applied in our management, the intraspinal surgical field was very small. We used 8-0 nylon suture to repair the defect of the cyst (Fig. 3F). The length of each defect varied and ranged from around 3 to $10 \mathrm{~mm}$. To effectively achieve dural closure, interrupted suture (for small defect) or running suture (for larger defect) was selectively performed according to the individualized situation, with delicate microsurgical manipulation under microscope. This technique had a high requirement for the surgical skill of the neurosurgeon. Supplementary dural substitute was not used, but muscle mud and fibrin glue were applied occasionally to reinforce the sutured defect when necessary. Marsupialization mainly applies to multiple-segments SEMC. When the defect is hard to identify and repair during surgery, connecting the large cyst and the subarachnoid space by extensively opening the inner layer of the cyst can stop one-way CSF collection in the cyst through the dural defect and result in a free and two-way flow of isotonic CSF. Even though decompression of the spinal cord and nerve roots are fully achieved when the cyst tension declines dramatically, a remarkably enlarged dural sac may still exist on the follow-up MRI after marsupialization ${ }^{10)}$. Simple aspiration of cyst liquid does not represent adequate treatment and should not be recommended ${ }^{2}$.

Most patients' symptoms are effectively relieved after surgery $^{3,5,23-25)}$. In this study, four patients became completely free of symptoms, while three reported substantial improvement. Three patients with a long interval of time before surgery were fair in outcome. The symptoms and neurological functions of No. 3 (muscle atrophy) and No. 5 (urination and defecation disturbance) patients remained stable postoperatively, but surgery did not cause any new pain or discomfort and they could still go back to regular work and life. The range of SEMC was extensive in patient No. 8 (T8 to L2). His spinal cord had been significantly compressed for more than 60 months and only a little symptomatic improvement was achieved after surgery. The possible causes for patients whose outcome were fair or poor include $e^{3,5,23)}:$ 1) long preoperative time interval; 2) extensive cyst range; and 3) severe sphincter dysfunction and/or muscle atrophy before surgery, which may result from longterm or widespread compression of the spinal cord and nerve roots by the cyst.

\section{Limitations}

This study has several limitations. First, SEMC is a rare disease, and the number of enrolled cases was not large. Second, this was a retrospective single-center study, and in two of 10 patients, we could not find clear radiological evidence of dural defects preoperatively. Third, there is a paucity of generally accepted methods, with high diagnostic accuracy and convenience, for the location of dural defect. Thus, an optimized preoperative assessment method is needed. Nonetheless, the current study may still provide valuable evidence for the surgical decision-making of SEMCs.

\section{CONCLUSION}

SEMCs are rare intraspinal-occupying cystic lesions. The formation of SEMC is proposed to be concerned with regional dural avulsion, and the cyst is further expanded by the hydrodynamic and pulsatile CSF forces. Radiography and MRI are effective methods for assessing the location of the defects. Selective hemilaminectomy or laminectomy can reduce surgical trauma and avoid postoperative kyphosis. The detection and microscopic suturing of the defects are the key steps to adequately decompress the nervous tissues and prevent postoperative recurrence.

\section{CONFLICTS OF INTEREST}

No potential conflict of interest relevant to this article was reported.

\section{INFORMED CONSENT}

Informed consent was obtained from all individual participants included in this study. 


\section{AUTHOR CONTRIBUTIONS}

\author{
Conceptualization : FX \\ Data curation : JG \\ Formal analysis : FX \\ Methodology : FJ \\ Project administration : ZC \\ Visualization : LL \\ Writing - original draft : FX \\ Writing - review \& editing : ZC
}

\section{ORCID}

$\begin{array}{ll}\text { Feifan Xu } & \text { https://orcid.org/0000-0003-0530-0654 } \\ \text { Fengzeng Jian } & \text { https://orcid.org/0000-0003-0862-7345 } \\ \text { Liang Li } & \text { https://orcid.org/0000-0001-9011-3233 } \\ \text { Jian Guan } & \text { https://orcid.org/0000-0001-6506-3844 } \\ \text { Zan Chen } & \text { https://orcid.org/0000-0002-7208-4149 }\end{array}$

\section{References}

1. Chen Z, Sun XL, Zhao Y, Wang K, Jian FZ : Dural dissection cyst: a more accurate term for extradural meningeal cyst. CNS Neurosci Ther 20 : 515-520, 2014

2. Choi JY, Kim SH, Lee WS, Sung KH : Spinal extradural arachnoid cyst. Acta Neurochir (Wien) $148:$ 579-585, 2006

3. Funao H, Nakamura M, Hosogane N, Watanabe K, Tsuji T, Ishii K, et al. : Surgical treatment of spinal extradural arachnoid cysts in the thoracolumbar spine. Neurosurgery 71 : 278-284, 2012

4. Furtado SV, Thakar S, Murthy GK, Dadlani R, Hegde AS : Management of complex giant spinal arachnoid cysts presenting with myelopathy. J Neurosurg Spine 15 : 107-112, 2011

5. Garg K, Borkar SA, Kale SS, Sharma BS : Spinal arachnoid cysts - our experience and review of literature. Br J Neurosurg 31 : 172-178, 2017

6. Gu K, Kwon JW, Kim ES : Digital subtraction cystography for detection of communicating holes of spinal extradural arachnoid cysts. Korean J Radiol 17 : 111-116, 2016

7. Hatashita S, Kondo A, Shimizu T, Kurosu A, Ueno H : Spinal extradural arachnoid cyst--case report. Neurol Med Chir (Tokyo) 41 : 318-321, 2001

8. Ishibe T, Senzoku F, Ikeda N, Kamba Y, Mikawa Y : Detection of the communicating hole(s) of spinal extradural arachnoid cysts using timespatial labeling inversion pulse magnetic resonance imaging. Spine (Phila Pa 1976) 39 : E1394-E1397, 2014

9. Ishibe T, Senzoku F, Kamba Y, Ikeda N, Mikawa Y : Time-spatial label- ing inversion pulse magnetic resonance imaging of cystic lesions of the spinal cord. World Neurosurg 88 : 693.e13-693.e21, 2016

10. Kikuta K, Hojo M, Gomi M, Hashimoto N, Nozaki K : Expansive duraplasty for the treatment of spinal extradural arachnoid cysts: case report. J Neurosurg Spine 4 : 251-255, 2006

11. Klekamp J : A new classification for pathologies of spinal meninges, part 1: dural cysts, dissections, and ectasias. Neurosurgery $81: 29-44$, 2017

12. Lee $\mathrm{CH}$, Hyun $\mathrm{SJ}$, Kim $\mathrm{KJ}$, Jahng TA, Kim HJ : What is a reasonable surgical procedure for spinal extradural arachnoid cysts: is cyst removal mandatory? Eight consecutive cases and a review of the literature. Acta Neurochir (Wien) 154 : 1219-1227, 2012

13. Lim MS, Khalil A, Okafo U, Dunlea O, Kaar G : Hemilaminectomy for large spinal extradural meningeal cysts: a case report and review of surgical techniques. Ann R Coll Surg Engl 98 : e162-e164, 2016

14. Liu JK, Cole CD, Kan P, Schmidt MH : Spinal extradural arachnoid cysts: clinical, radiological, and surgical features. Neurosurg Focus 22 : E6, 2007

15. Millward CP, Bhagawati D, Chan HW, Bestwick J, Brecknell JE : Retrospective observational comparative study of hemilaminectomy versus laminectomy for intraspinal tumour resection; shorter stays, lower analgesic usage and less kyphotic deformity. Br J Neurosurg 29 : 390 395, 2015

16. Mishra RK, Pruthi N, Bharath RD, Malla BR : Role of intraoperative dynamic magnetic resonance myelogram in management of giant dorsolumbar spinal extradural arachnoid cyst: case report. J Neurosurg Spine 27 : 185-188, 2017

17. Miyakoshi N, Hongo M, Kasukawa Y, Shimada Y : Huge thoracolumbar extradural arachnoid cyst excised by recapping T-saw laminoplasty. Spine J 10 : e14-e18, 2010

18. Miyamoto M, Kim K, Matsumoto R, Isobe M, Isu T : Utility of preoperative magnetic resonance imaging myelography for identifying dural defects in patients with spinal extradural arachnoid cysts: case report. Neurosurgery 59 : E941, 2006

19. Morizane K, Fujibayashi S, Otsuki B, Sakamoto T, Tsutsumi R, Odate S, et al. : Clinical and radiological features of spinal extradural arachnoid cysts: valve-like mechanism involving the nerve root fiber as a possible cause of cyst expansion. J Orthop Sci 23 : 464-469, 2018

20. Nabors MW, Pait TG, Byrd EB, Karim NO, Davis DO, Kobrine Al, et al. : Updated assessment and current classification of spinal meningeal cysts. J Neurosurg 68 : 366-377, 1988

21. Neo M, Koyama T, Sakamoto T, Fujibayashi S, Nakamura T : Detection of a dural defect by cinematic magnetic resonance imaging and its selective closure as a treatment for a spinal extradural arachnoid cyst. Spine (Phila Pa 1976) 29 : E426-E430, 2004

22. Netra R, Min L, Shao Hui M, Wang JC, Bin Y, Ming Z : Spinal extradural meningeal cysts: an MRI evaluation of a case series and literature review. J Spinal Disord Tech 24 : 132-136, 2011

23. Oh JK, Lee DY, Kim TY, Yi S, Ha Y, Kim KN, et al. : Thoracolumbar extradural arachnoid cysts: a study of 14 consecutive cases. Acta Neurochir $154: 341-348,2012$ 
24. Qi W, Zhao L, Fang J, Chang $X, X u Y$ : Clinical characteristics and treatment strategies for idiopathic spinal extradural arachnoid cysts: a singlecenter experience. Acta Neurochir (Wien) 157 : 539-545, 2015

25. Tokmak M, Ozek E, Iplikcioglu AC : Spinal extradural arachnoid cysts: a series of 10 cases. J Neurol Surg A Cent Eur Neurosurg 76 : 348-
352,2015

26. Tureyen K, Senol N, Sahin B, Karahan N : Spinal extradural arachnoid cyst. Spine J 9 : e10-e15, 2009

27. Woo JB, Son DW, Kang KT, Lee JS, Song GS, Sung SK, et al. : Spinal extradural arachnoid cyst. Korean J Neurotrauma 12 : 185-190, 2016 